\title{
Un método para el diseño óptimo de redes de distribución de agua potable
}

\author{
Ó. A. Fuentes Mariles y J. J. Carrillo Sosa \\ Instituto de Ingeniería, UNAM \\ Email: ofm@pumas.iingen.unam.mx
}

(recibido: abril, 1997; aceptado: febrero, 1998)

\section{Resumen}

Se presenta un método para seleccionar, de entre varias opciones de diámetros comerciales, la combinación que forma la red de tuberías más económica que satisface las condiciones de gasto en los tubos y de presión en los nudos de la misma. Para el análisis hidráulico se emplea un método estático del Instituto de Ingeniería, UNAM y se aplica una técnica de optimación para reducir el número de cálculos y alcanzar la solución.

\begin{abstract}
A method to select, among several commercial diameter options, the most economic combination of pipeline networks is presented. These networks comply with the discharge conditions in the pipes and pressure in the knots. It is applied a static method for the hydraulics analysis (Institute of Engineering, UNAM) and an optimization technique to reduce the number of calculations.
\end{abstract}

\section{Introducción}

La revisión, operación y diseño de redes de tuberías empleadas en el suministro de agua se basa en las ecuaciones fundamentales del movimiento de fluidos a presión.

La revisión hidráulica consiste en determinar los gastos que circulan en las tuberías y los niveles piezométricos (cargas de presión) en varios puntos de la red. Para ello, se necesita conocer las características físicas de las tuberías, sus conexiones, gastos de demanda, elevaciones de tanques reguladores, etc.

La operación se enfoca a las maniobras de apertura y cierre de válvulas, control de niveles en tanques, y arraque y paro de bombas para disponer de cargas de presión adecuadas en la red.

El diseño hidráulico se refiere a la selección de los diámetros de las tuberías que forman la red para conducir el fluido hasta los sitios de demanda de modo tal que las presiones sean adecuadas. La presión en cualquier punto de la red debe ser mayor a una mínima $\left(h_{\min }\right)$ para que el agua llegue a los domicilios, y menor a una máxima $\left(h_{\text {maxx }}\right)$ para evitar la rotura de tuberías y gastos de fugas excesivos. Se recomienda que estas presiones extremas sean de $10 \mathrm{~m}$ y $30 \mathrm{~m}^{2}$ respectivamente ${ }^{1}$.

El proceso de selección de los diámetros de las tuberías de la red no es simple, ya que para llevar el agua a los sitios de consumo existen numerosas opciones que satisfacen las condiciones de operación hidráulica, por lo que, para escoger la opción que tiene el mínimo presupuesto de adquisición e instalación es necesario conocer el costo de todas las opciones que cumplen con las condiciones hidráulicas.

En el diseño de la red de tuberías es importante su trazo; éste consiste en la unión de los puntos de demanda por medio de líneas, de modo tal que sigan la configuración urbana y la topografía de la zona. En general, ese trazo se define formando circuitos y atendiendo a criterios de carácter no hidráulico, por lo cual no suele ser incluido dentro de los métodos de diseño de redes.

En virtud de que en las ecuaciones de pérdida de carga por fricción entre los extremos de cada tubería de una red aparecen el gasto elevado al cuadrado y el diámetro a un exponente distinto de uno (ambos son desconocidos en el diseño de redes), se han planteado distintos tipos de métodos; en algunos, se fija el valor de los gastos y se considera como incógnitas a los diámetros; en otros, se propone valores a los diámetros de las tuberías de la red y se verifica que cumplan con las restricciones de operación.

En los primeros métodos de diseño se impuso que la suma de las longitudes de los conductos que unen a los puntos de demanda de la red fuera mínima; sin embargo, la red se determina una vez que han sido escogidos losdiámetros de

\footnotetext{
${ }_{1}^{1}$ Manual de diseño de agua potable, alcantarillado y saneamiento, "Redes de distribución", Comisión Nacional del Agua, 1996.
} 
las tuberías de acuerdo con la experiencia del diseñador (Tong, 1961). En 1966, Raman y Raman propusieron modificar el método anterior condicionando a que en los circuitos, la longitud mínima de tubería se encontrara cuando se cumpliera que la suma de las longitudes entre el gasto fuera nula. Con este método tampoco se encontraba propiamente un diseño económico.

Deb y Sarkar (1971) plantearon minimizar los diámetros utilizados por medio de una ecuación de pérdida de energía junto con una función lineal del costo de la tubería. Un método similar fue empleado por Watanatada (1973), quien empleó un sistema de programación no lineal y luego redondeó los resultados a valores de diámetros comerciales; sin embargo, ello no garantiza que la solución sea la óptima, ya que al ajustar los diámetros, cambian las condiciones de optimación empleadas y varían las condiciones hidráulicas.

Con base en los primeros modelos, se propusieron procedimientos en los que se establece una función objetivo sujeta a varias restricciones basadas en el trazo de la red, la condición de operación hidráulica y los diámetros comerciales.

Uno de los métodos de optimación de redes más conocido es el de Alperovits y Shamir (1977); en él se plantea como función objetivo la suma de los costos de cada tubería. El costo total de la tubería se obtiene al sumar los productos del costo por metro, lineal asociado a un cierto diámetro, por la longitud del tramo. Este método busca minimizar la función, que tenía la virtud de ser lineal.

En las restricciones planteadas se establece que la suma algebraica de las pérdidas de carga en un circuito sea igual a cero. Por otro lado, las incógnitas a determinar corresponden a las longitudes de los tramos de cierto diámetro consideradas en cada tubería de la red.

Este método tiene la desventaja de que en cada tubería deben existir más de dos tramos con diámetros diferentes, lo cual no es práctico desde el punto de vista constructivo. También, requiere establecer de antemano la dirección y la magnitud de los gastos por conducir en cada tubería y esto no asegura una solución óptima.

\section{Método de diseño óptimo propuesto}

Se presenta un método de optimación de redes que no requiere una distribución inicial de los gastos en las tuberías de la red (se elimina una de las limitaciones de otros métodos). Además, los diámetros seleccionados para formar la red son comerciales.

\section{Descripción del método}

Se entenderá como cálculo de la red la obtención de los gastos que circulan en las tuberías y las presiones en sus nudos para flujo permanente. Se recomienda obtenerlo por el método de red estática (Fuentes y Sánchez, 1991).

El costo de la red se calcula por medio de la expresión siguiente:

$$
C=\sum_{j=1}^{N} C_{i} L_{j},
$$

donde:

$\mathrm{L}_{j}$ es la longitud de la tubería $j$

$\mathrm{C}_{i}$ es el costo por metro lineal de la tubería

que tiene el diámetro comercial $D_{i}$

$\mathrm{N}$ es el número de tuberías de la red

Se considera que los gastos de salida de la red corresponden a la máxima demanda horaria. ${ }^{2}$

\section{Arreglo de diámetros comerciales disponibles}

Se calcula la red considerando que todas sus tuberías tienen el mismo diámetro y que éste es igual al más pequeño de entre los comercialmente posibles $\left(D_{\min }\right)$. Se revisa que las presiones calculadas cumplan con las restricciones de presión. En el caso, poco frecuente, de que sí las satisfagan, el diseño óptimo consistiría en esta selección de diámetros y la aplicación del método concluye. De otro modo, se propone al diámetro comercial inmediato superior para todas las tuberías de la red y se calcula la red de tubos. Se revisa que se cumplan las restricciones de presión y se continúa el proceso hasta que se haya escogido para la red el diámetro económico que implica que se logren las restricciones de presión. De esta manera, la red tendría en todas sus tuberías el diámetro comercial que será denominado $D_{\max }$.

El arreglo de diámetros comerciales disponible estará formado por todos los diámetros comprendidos entre $D_{\min } \mathrm{Y}$ $D_{\text {max }}$ inclusive.

\section{Costo máximo y mínimo de la red}

Mediante la ecuación 1 se obtiene el costo de la red $C_{\text {min }}$ para el caso en que todas las tuberías tengan el diámetro $D_{\min }$ y el costo de la red $C_{\text {máx }}$ que tiene diámetro $\mathrm{D}_{\max }$ en todas ellas.

\section{Combinaciones de diámetros para las tuberías de la red asociadas al costo $C_{p}$}

Se propone un costo $C_{p}$ para la red de tuberías comprendido entre $C_{\min }$ y $C_{\max }$, y se escoge para cada tubería un diámetro entre los incluidos en el arreglo de diámetros comerciales disponibles, de modo que el costo obtenido con la ecuación 1 sea del orden de $C_{p}$. Por tanto, se forman

\footnotetext{
2 Se empleó la curva de variación horaria de la demanda deducida en el Instituto de Ingeniería, UNAM.
} 
varios conjuntos que constituyen, cada uno de ellos, una combinación factible de diámetros.

\section{Revisión hidráulica}

Para cada una de las combinaciones factibles se calcula la red; esto se hace de forma expedita por medio del método estático del Instituto de Ingeniería (Fuentes y Sánchez, 1991). Luego se revisa que todos sus nudos cumplan con las restricciones de presión. Cuando varias combinaciones cumplen con estas restricciones, se escoge la de costo mínimo (cercano a $C_{p}$ ).

El método implica escoger el costo propuesto $C_{p}$ más pequeño posible para el cual existe al menos una combinación que cumpla con las restricciones. Este costo puede ser encontrado mediante técnicas de búsqueda secuencial de optimación.

Las técnicas de búsqueda secuencial empiezan con un intervalo finito dentro del cual la función obje tivo tiene un solo punto donde es máxima o mínima. En cada iteración se disminuye el intervalo dentro del cual existe dicho punto; el tamaño del intervalo debe ser menor al de un valor especificado de antemano.

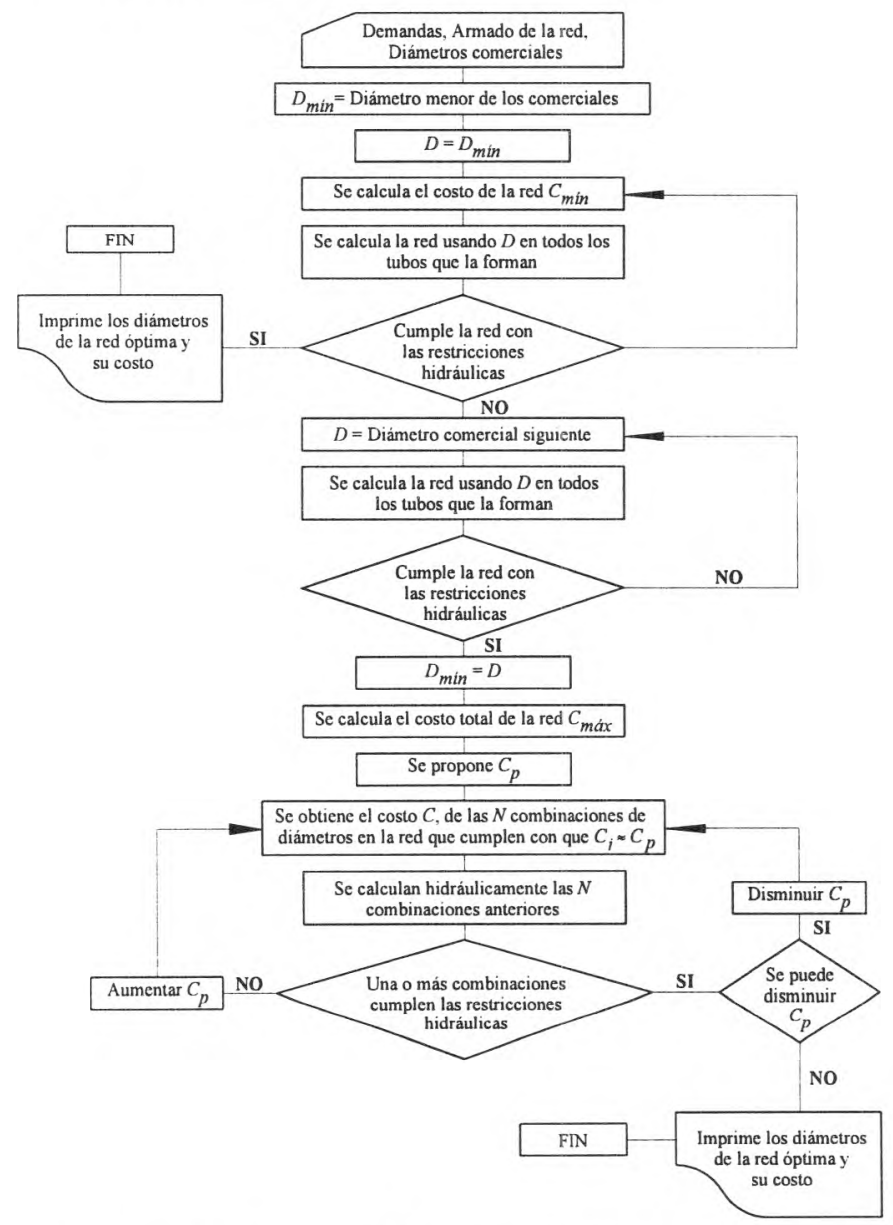

Figura 1. Diagrama del flujo del método de diseño óptimo
Una de las técnicas secuenciales que se recomienda emplear es la de Fibonacci, la cual se basa en el número $\left(5^{1 / 2}, 1\right) / 2=0.618$, que da lugar a la llamada regla de oro.

En la figura 1 se presenta el diagrama de flujo del método de diseño de redes.

\section{Ejemplos de aplicación}

En la figura 2 y la tabla 1 se presenta un ejemplo de una red en la que se ha definido el trazo y se conocen los gastos de la demanda máxima horaria en los nudos. Se aplicará el método para encontrar los diámetros que deben formar la red y la hagan funcionar adecuadamente, además de que su costo sea el mínimo.

En la tabla 2 se presentan los costos para tubería de asbesto-cemento clase A-5 por el diámetro, actualizados a julio de 1996. Incluyen instalación, suministro y piezas especiales.

El método descrito se aplica empleando la técnica secuencial de Fibonacci.

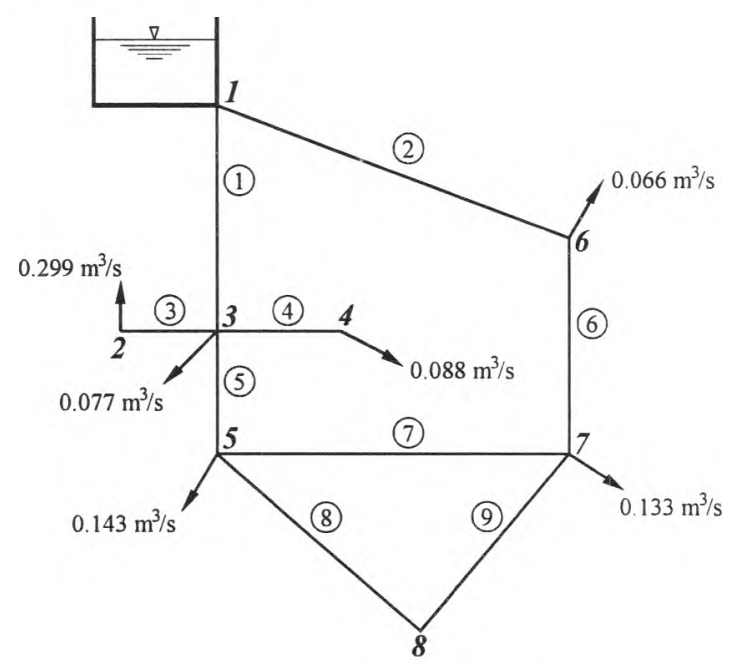

Figura 2. Red ejemplo

Tabla 1. Características de la red

\begin{tabular}{ccccc}
\hline \hline Tubo & Longitud $(\mathrm{m})$ & Nudo & $Q_{\mathrm{dem}}\left(\mathrm{m}^{3} \mathrm{~s}\right)$ & Cota $(\mathrm{msmn})$ \\
\hline \hline 1 & 5205 & 1 & 0.000 & 2314.80 \\
2 & 4195 & 2 & 0.299 & 2241.40 \\
3 & 350 & 3 & 0.077 & 2241.40 \\
4 & 995 & 4 & 0.088 & 2238.00 \\
5 & 1760 & 5 & 0.143 & 2237.60 \\
6 & 2310 & 6 & 0.066 & 2238.00 \\
7 & 1740 & 7 & 0.133 & 2237.50 \\
8 & 1000 & 8 & 0.000 & 2237.55 \\
9 & 1150 & & & \\
\hline \hline
\end{tabular}


Tabla 2. Costos de tuberías de asbesto-cemento, clase A-5

\begin{tabular}{|c|c|c|}
\hline \multicolumn{2}{|c|}{ Diámetro } & \multirow[b]{2}{*}{ Costo $(\$ / \mathrm{m})$} \\
\hline Milímetros & Pulgadas & \\
\hline 50 & 2 & 10.834 \\
\hline 100 & 4 & 26.677 \\
\hline 150 & 6 & 45.191 \\
\hline 200 & 8 & 65.686 \\
\hline 250 & 10 & 87.792 \\
\hline 300 & 12 & 111.273 \\
\hline 350 & 14 & 135.962 \\
\hline 400 & 16 & 161.737 \\
\hline 450 & 18 & 188.498 \\
\hline 500 & 20 & 216.168 \\
\hline 600 & 24 & 273.985 \\
\hline
\end{tabular}

Los diámetros factibles a ser empleados en las redes de distribución van desde seis hasta veinticuatro plg de diámetro (tabla 3).
Tabla 3. Diámetros factibles

\begin{tabular}{lrrrrrrrr}
\hline \hline Diámetro & 1 & 2 & 3 & 4 & 5 & 6 & 7 & 8 \\
\hline$(\mathrm{mm})$ & 150 & 200 & 250 & 300 & 350 & 400 & 450 & 500 \\
$(\mathrm{plg})$ & 6 & 8 & 10 & 12 & 14 & 16 & 18 & 204 \\
\hline \hline
\end{tabular}

Al emplear el diámetro mínimo en toda la red del ejemplo no se obtienen resultados que satisfagan las condiciones hidráulicas (tabla 4).

De acuerdo con lo indicado anteriormente, se calcula la red aumentando el diámetro hasta que se cumple con las condiciones hidráulicas, usando el mismo diámetro en todas las tuberías. Esto se logra usando el diámetro de dieciséis plg, tal como se observa en la tabla 5. Al aplicar el procedimiento descrito, se llega a la red óptima en 16 iteraciones (tabla 6); el funcionamiento hidráulico de esta red es mostrado en la tabla 7.

Tabla 4. Resultados usando el diámetro de seis pulgadas $\left(D_{\text {min }}\right)$

\begin{tabular}{|c|c|c|c|c|c|c|}
\hline $\begin{array}{l}\text { Tubo } \\
\text { No. }\end{array}$ & $\begin{array}{c}\text { Gasto } \\
(1 / s)\end{array}$ & $\begin{array}{l}\text { Sale del } \\
\text { Nudo }\end{array}$ & Nudo & $\begin{array}{c}\text { Nivel } \\
\text { Piezométrico (m) }\end{array}$ & $\begin{array}{l}\text { Carga sobre } \\
\text { terreno }(\mathrm{m})\end{array}$ & $\begin{array}{l}\text { Gasto de demanda } \\
\qquad(1 / s)\end{array}$ \\
\hline 1 & 375.70 & 1 & 1 & 2316.80 & 2.00 & 0 \\
\hline 2 & 353.15 & 1 & 2 & -6215.70 & -4009.30 & 299 \\
\hline 3 & 298.99 & 3 & 3 & -5860.44 & -3619.04 & 77 \\
\hline 4 & 88.000 & 3 & 4 & -5961.34 & -3723.34 & 88 \\
\hline 5 & 5.357 & 3 & 5 & -5860.34 & -3622.74 & 143 \\
\hline 6 & 287.15 & 6 & 6 & .3536 .72 & -1298.72 & 66 \\
\hline 7 & 81.467 & 7 & 7 & .5706 .45 & -3468.96 & 133 \\
\hline 8 & 72.689 & 8 & 8 & -5789.233 & -3551.68 & 0 \\
\hline 9 & 72.690 & 7 & & & -13.3768 & \\
\hline
\end{tabular}

Tabla 5. Resultados usando el diámetro de dieciséis pulgadas $\left(D_{\max }\right)$

\begin{tabular}{|c|c|c|c|c|c|c|}
\hline $\begin{array}{l}\text { Tubo } \\
\text { No. }\end{array}$ & $\begin{array}{c}\text { Gasto } \\
(\mathrm{l} / \mathrm{s})\end{array}$ & $\begin{array}{l}\text { Sale del } \\
\text { Nudo }\end{array}$ & Nudo & $\begin{array}{c}\text { Nivel } \\
\text { piezométrico }(\mathrm{m})\end{array}$ & $\begin{array}{l}\text { Carga sobre } \\
\text { terreno }(\mathrm{m})\end{array}$ & $\begin{array}{c}\text { Gasto de demanda } \\
(1 / \mathrm{s})\end{array}$ \\
\hline 1 & 364.21 & 1 & 1 & 2316.80 & 2.00 & 0 \\
\hline 2 & 335.30 & 1 & 2 & 2251.43 & 10.03 & 299 \\
\hline 3 & 299.13 & 3 & 3 & 2253.30 & 11.90 & 77 \\
\hline 4 & 87.984 & 3 & 4 & 2252.43 & 14.43 & 88 \\
\hline 5 & 10.348 & 3 & 5 & 2253.33 & 15.74 & 143 \\
\hline 6 & 286.61 & 6 & 6 & 2272.17 & 34.17 & 66 \\
\hline 7 & 81.255 & 7 & 7 & 2254.66 & 17.16 & 133 \\
\hline 8 & 72.310 & 8 & 8 & 2253.95 & 16.40 & 0 \\
\hline 9 & 72.270 & 7 & & & & \\
\hline
\end{tabular}


Tabla 6. Resultados del método de diseño óptimo de redes

\begin{tabular}{cccccccccc}
\hline & \multicolumn{7}{c}{ Diámetro de las tuberías que forman la red (en pulgadas) } \\
\cline { 2 - 8 } Tubería & $\mathrm{F}=0.618$ & $\mathrm{~F}=0.382$ & $\mathrm{~F}=0.5278$ & $\mathrm{~F}=0.4721$ & $\mathrm{~F}=0.4376$ & $\mathrm{~F}=0.4164$ & $\mathrm{~F}=0.4032$ & $\mathrm{~F}=0.3951$ \\
\hline 9 & 10 & $\ldots$ & 8 & 6 & 6 & 6 & 6 & 6 \\
8 & 12 & $\ldots$ & 10 & 8 & 6 & 6 & 6 & 6 \\
3 & 16 & $\ldots$ & 14 & 16 & 14 & 14 & 16 & 16 \\
4 & 14 & $\ldots$ & 14 & 12 & 14 & 12 & 10 & 12 \\
5 & 8 & $\ldots$ & 10 & 8 & 10 & 8 & 10 & 10 \\
6 & 16 & $\ldots$ & 14 & 16 & 16 & 16 & 16 & 14 \\
7 & 14 & $\ldots$ & 14 & 16 & 14 & 16 & 14 & 14 \\
2 & 16 & $\ldots$ & 16 & 16 & 16 & 16 & 16 & 16 \\
Costo propuesto & 12 & $\ldots$ & 14 & 16 & 16 & 14 & 16 & 16 \\
Costo combinación & 2192533 & 1677889 & 1995939 & 1874444 & 1799360 & 1752958 & 1724282 & 1706560 \\
\hline
\end{tabular}

continúa...

Tabla 6. Resultados del método de diseño óptimo de redes (continuación)

\begin{tabular}{ccccccccc}
\hline & \multicolumn{7}{c}{ Diámetro de las tuberias que forman la red (en pulgadas) } \\
\cline { 2 - 9 } Tubería & $\mathrm{F}=0.3901$ & $\mathrm{~F}=0.3869$ & $\mathrm{~F}=0.3850$ & $\mathrm{~F}=0.3838$ & $\mathrm{~F}=0.3831$ & $\mathrm{~F}=0.3827$ & $\mathrm{~F}=0.3824$ & $\mathrm{~F}=0.3822$ \\
\hline $\mathrm{S}$ & 6 & 6 & 6 & 6 & 6 & 6 & 6 & 6 \\
8 & 6 & 6 & 6 & 6 & 6 & 6 & 6 & 6 \\
3 & 14 & 12 & 12 & 12 & 12 & 12 & 14 & 14 \\
4 & 14 & 12 & 10 & 12 & 14 & 12 & 10 & 10 \\
5 & 12 & 10 & 8 & 8 & 6 & 6 & 8 & 8 \\
6 & 14 & 14 & 16 & 16 & 16 & 16 & 14 & 14 \\
7 & 12 & 14 & 14 & 12 & 12 & 14 & 16 & 16 \\
2 & 16 & 16 & 16 & 16 & 16 & 16 & 16 & 16 \\
1 & 14 & 16 & 16 & 16 & 14 & 14 & 16 & 16 \\
Costo propuesto & 1695608 & 1688839 & 1684656 & 1682071 & 1680474 & 1679487 & 1678876 & 1678499 \\
Costo combinación & 1652039 & 1645315 & 1641533 & 1639901 & 1637186 & 1636692 & 1635483 & 1635483 \\
\hline
\end{tabular}

Red óptima $\uparrow$

Tabla 7. Resultados del cálculo hidráulico de la red óptima

\begin{tabular}{|c|c|c|c|c|c|c|c|}
\hline $\begin{array}{l}\text { Tubo } \\
\text { No. }\end{array}$ & Gasto $(\mathrm{l} / \mathrm{s})$ & $\begin{array}{l}\text { Sale del } \\
\text { Nudo }\end{array}$ & $\mathrm{HF}(\mathrm{m})$ & Nudo & $\begin{array}{c}\text { Nivel } \\
\text { piezométrico }(\mathrm{m})\end{array}$ & $\begin{array}{c}\text { Carga sobre terreno } \\
(\mathrm{m})\end{array}$ & $\begin{array}{c}\text { Gasto de demanda } \\
(1 / \mathrm{s})\end{array}$ \\
\hline 9 & 3.64 & 7 & 1.383 & 8 & 2265.412 & 27.862 & 0 \\
\hline 8 & 3.54 & 8 & 1.056 & 6 & 2303.424 & 65.424 & 66 \\
\hline 3 & 298.8 & 3 & 9.082 & 3 & 2272.881 & 31.481 & 77 \\
\hline 4 & 88.01 & 3 & 21.307 & 2 & 2263.800 & 22.400 & 299 \\
\hline 5 & 42.38 & 3 & 8.525 & 4 & 2251.574 & 13.574 & 88 \\
\hline 6 & 233.58 & 6 & 36.630 & 5 & 2264.356 & 26.756 & 143 \\
\hline 7 & 96.97 & 7 & 2.439 & 7 & 2266.794 & 29.292 & 133 \\
\hline 1 & 506.16 & 1 & 43.919 & 1 & 2316.800 & 2.00 & 0 \\
\hline 2 & 299.55 & 1 & 13.376 & & & & \\
\hline
\end{tabular}


Tabla 8. Resultados del método de Alperovits y Shamir

\begin{tabular}{|c|c|c|c|c|c|c|c|}
\hline Tubo & $\mathrm{L} 1(\mathrm{~m})$ & D1 (plg) & $\mathrm{L} 2(\mathrm{~m})$ & D2 (plg) & Nudo & Carga (m) & Costo \\
\hline 1 & 0.00 & 0 & 4195.00 & 24 & 1 & 2.00 & 1149368.41 \\
\hline 2 & 1542.00 & 16 & 3663.00 & 14 & 2 & 10.00 & 747428.41 \\
\hline 3 & 114.34 & 14 & 235.66 & 12 & 3 & 32.70 & 41768.43 \\
\hline 4 & 595.32 & 12 & 1164.68 & 10 & 4 & 10.00 & 168491.98 \\
\hline 5 & 0.00 & 0 & 995.00 & 14 & 5 & 22.80 & 135282.68 \\
\hline 6 & 0.00 & 0 & 2310.00 & 12 & 6 & 29.20 & 257039.74 \\
\hline 7 & 1685.74 & 6 & 54.26 & 4 & 7 & 10.00 & 77627.33 \\
\hline 8 & 1150.00 & 8 & 0.00 & 0 & 8 & 16.40 & 75548.90 \\
\hline \multirow[t]{2}{*}{9} & 862.00 & 8 & 138.00 & 6 & & & 62855.88 \\
\hline & \multicolumn{6}{|c|}{ Costo total de la red } & 2715411.83 \\
\hline
\end{tabular}

La misma red fue calculada por el método de Alperovits y Shamir (1977). En este caso, se planteó que en cada tubería que forma la red se podría contar hasta con tres posibles diámetros candidatos. Los diámetros comerciales de cada tubo pueden ser candidatos para desventaja del método. En la tabla 8 se presenta un resumen de los resultados obtenidos por este método para el mismo ejemplo; en ella se observa que existen tramos de la red que se forman por dos diámetros diferentes. El costo total de la solución usando el método de Alperovits y Shamir es de 2715 412, mayor de 1635 483, que es el costo de la solución obtenida con el método propuesto; para formar parte de ese tramo son elegidos a criterio del proyectista, lo cual no garantiza la solución óptima. De igual manera, la distribución de agua en la red se debe determinar previamente, lo que representa otra desventaja.

\section{Conclusiones}

El método propuesto para el diseño óptimo de redes de tuberías permite encontrar la combinación de tubos con el costo más bajo de entre un conjunto de combinaciones posibles acordes con los diámetros comerciales factibles. Tiene la ventaja de que la distribución del agua en las tuberías no necesita determinarse previamente. Su aplicación es sencilla y el análisis hidráulico de las distintas combinaciones seleccionadas durante la misma es ágil al emplear el método estático para análisis de redes del Instituto de Ingeniería.

\section{Referencias}

Alperovits E. y Shamir U. (1977). Desing of Optimal Water Distribution Systems. Water Resources Research, Vol. 13, No. 6.

Deb y Sarkar A. (1971). Optimation in Desing of Hydraulics Network. Journal of the Sanitary Engineering Division, Proceedings of the American Society of Civil Engineerings, Vol. 97, No. SA2.

Fuentes O. y Sánchez J.L. (1991). Actualización de un método para calcular redes de tuberías funcionando en régimen permanente, Ingeniería, Vol. LXI, No. 4

Raman V. y Raman S. (1966). New Method of solving Distribution System Network Based on Equivalent Pipe Lengths. Journal of the American Water Works Association, mayo.

Tong, A. et al (1961). Analysis of Distribution Network by Balancing Equivalent Pipe Lengths, Journal of the $A W W A$, enero.

Watanatada P. (1973). Least-Cost desing of Water Distribution Systems. Journal of Hydraulics Division, ASCE, noviembre.

\section{Semblanza de los autores}

Óscar Arturo Fuentes Mariles. Ingeniero civil egresado de la Facultad de Ingeniería, UNAM. Realizó estudios de maestría y doctorado en ingeniería hidráulica en la División de Estudios de Posgrado de la misma Facultad; actualmente imparte los cursos de métodos numéricos y mecánica de fluidos. Es investigador del Instituto de Ingeniería, UNAM y jefe del área de Riesgos Hidrometeorológicos en el Centro Nacional de Prevención de Desastres de la Secretaría de Gobernación. Ha colaborado en más de 50 proyectos de investigación y tiene más de 80 publicaciones científicas relacionadas con hidráulica marítima, hidrología e hidráulica urbana.

Juan Javier Carrillo Sosa. Ingeniero civil egresado de la Escuela Nacional de Estudios Profesionales Aragón, UNAM. Realizó estudios de maestría en hidráulica en la División de Estudios de Posgrado de la Facultad de Ingeniería. Desde 1997, es profesor en el laboratorio de hidráulica en la Universidad Autónoma Metropolitana. Su experiencia en consultoría privada se relaciona con proyectos de agua potable, alcantarillado y saneamiento. Actualmente, es técnico académico en el Instituto de Ingeniería UNAM, donde ha participado en proyectos de investigación relacionados con el análisis y diseño de redes de conducción de agua funcionando a presión, y flujo en superficie libre. 